\title{
Choosing between Enoxaparin and Fondaparinux for the management of patients with acute coronary syndrome: $A$ systematic review and meta-analysis
}

\author{
Pravesh Kumar Bundhun ${ }^{1}$, Musaben Shaik ${ }^{2}$ and Jun Yuan ${ }^{3^{*}}$ (i)
}

\begin{abstract}
Background: Enoxaparin and Fondaparinux are potential anticoagulants which are used peri-operatively in the management of patients with Acute Coronary Syndrome (ACS). We aimed to compare the adverse clinical outcomes which are associated with the use of these anticoagulants in patients who were treated for ACS.

Methods: Online databases (PubMed/Medline, EMBASE, Cochrane library) were searched for studies which compared differences in clinical outcomes observed with the use of enoxaparin and fondaparinux in patients who were treated peri-operatively for ACS. Statistical analysis was carried out by Revman 5.3 software with odds ratio (OR) and $95 \%$ confidence intervals $(\mathrm{Cl})$ as the analytical parameters.

Results: Seven studies with a total number of 9618 patients (mainly composed of non-ST elevated myocardial infarction/NSTEMI) were included. This analysis showed mortality to be similarly observed between enoxaparin and fondaparinux with OR: 1.05, 95\% Cl: 0.67-1.63; $P=0.84$. Myocardial infarction (MI) and stroke were also not significantly different throughout different follow up periods. However, minor, major and total bleeding were significantly lower with fondaparinux (OR: 0.40, 95\% Cl: 0.27-0.58; $P=0.00001)$, (OR: 0.46, 95\% Cl: 0.32-0.66; $P=0$. $0001)$ and (OR: $0.47,95 \% \mathrm{Cl}: 0.37-0.60 ; P=0.00001$ ) respectively during the 10-day follow up period. Even during a follow up period of 30 days or a midterm follow up, major and minor bleeding still significantly favored fondaparinux in comparison to enoxaparin.
\end{abstract}

Conclusion: In patients who were treated for ACS, fondaparinux might be a better choice when compared to enoxaparin in terms of short to midterm bleeding events. This result was mainly applicable to patients with NSTEMI. However, due to a limited number of patients analyzed, further larger randomized trials should be able to confirm this hypothesis.

Keywords: Enoxaparin, Fondaparinux, Acute coronary syndrome, Major bleeding, Minor bleeding, Heparin

\section{Background}

Enoxaparin and Fondaparinux are potential anticoagulants which are used peri-operatively in the management of patients with Acute Coronary Syndrome (ACS) [1]. The Fifth Organization to Assess Strategies in Ischemic Syndromes (OASIS 5) trial showed fondaparinux to reduce the rate of major bleeding and net clinical benefit including

\footnotetext{
* Correspondence: nnyuanjun@163.com

${ }^{3}$ Department of Cardiology, The People's Hospital of Guangxi Zhuang

Autonomous Region, Nanning, Guangxi 530021, China

Full list of author information is available at the end of the article
}

death, Myocardial Infarction (MI), stroke and major bleeding in comparison to enoxaparin [2]. However, results from the French Registry of ST segment elevation and non-ST segment elevation MI (FAST-MI) 2010 showed a similar rate of bleeding and mortality between fondaparinux and enoxaparin [3]. Even though, FAST-MI cannot be compared to the OASIS 5 trial which consisted of a very large number of patients, we would still like to confirm the results through a meta-analysis by comparing the adverse clinical outcomes which were observed with enoxaparin and fondaparinux in patients who were treated for ACS. 


\section{Methods}

\section{Data sources and search strategy}

Online databases (PubMed/Medline, EMBASE, Cochrane library) were searched for studies (English publications) which compared differences in clinical outcomes observed with the use of enoxaparin and fondaparinux in patients who were treated peri-operatively for ACS by using the searched terms 'enoxaparin and fondaparinux and acute coronary syndrome'. Later in this search process, the terms 'percutaneous coronary intervention, heparin' and the abbreviations 'ACS and PCI' were also used. Reference lists of suitable articles were also reviewed for relevant publications.

\section{Inclusion criteria}

Studies were included if they satisfied certain major criteria which were:

(a) They were randomized controlled trials or observational studies comparing enoxaparin with fondaparinux in patients who were treated for ACS or in patients who were undergoing percutaneous coronary intervention (PCI).

(b) They reported adverse outcomes (cardiovascular and bleeding outcomes) as their clinical endpoints.

(c) They involved relevant data which could be used in this current analysis.

\section{Exclusion criteria}

Studies were excluded if they did not satisfy certain major criteria which were:

(a) They were systematic reviews, meta analyses, case studies or letter to editors.

(b) They did not include patients with ACS.

(c) They did not report the previously mentioned clinical outcomes.

(d)They were duplicates of the same study or they were associated with the same trial or cohort.

\section{Types of participants}

This analysis mainly included patients with non-ST segment elevated myocardial infarction (NSTEMI), patients with unstable angina (UA) and a small percentage of patients with ST segment elevated myocardial infarction (STEMI).

\section{Definitions, outcomes and follow ups}

Endpoints which were assessed included:
(a) Mortality;
(b) MI;
(c) Stroke;
(d)Minor bleeding: any type of minor bleeding;
(e) Major bleeding: any type of major bleeding;
(f) All bleeding (major and minor bleeding combined) including thrombolysis in myocardial infarction (TIMI) defined major and minor bleeding [4].

The follow up periods were classified as:

- Less than 10 days (including in-hospital follow up period);

- 30 days follow up period;

- Mid-term follow up (6 months to 1 year) period.

These outcomes and follow up periods have been summarized in Table 1.

\section{Data extraction and review}

Information and data including the name of authors, year of article publication, period of patients' enrollment, number of patients collected from each group (enoxaparin and fondaparinux), type of study (RCT or observational study), baseline characteristics of the patients, the outcomes reported, the follow up periods, the medications used by the patients, and the number of events reported with enoxaparin and fondaparinux respectively, were independently collected/extracted by two authors (PKB and MS). Disagreements were resolved by discussion with the third author (JY). Since this is a meta-

Table 1 Reported Outcomes and follow up periods

\begin{tabular}{|c|c|c|c|}
\hline Studies & Reported outcomes & Follow up period & Types of participants \\
\hline FAST MI [3] & Death, MI, stroke, TIMI major and minor bleeding & In-hospital & NSTEMI \\
\hline OASIS 5 [2] & Death, MI, stroke, TIMI major bleeding, total bleeding, major bleeding & 9 days, 30 days, 6 months & NSTEMI, UA \\
\hline Schiele 2010 [14] & Death, bleeding & 30 days & STEMI, NSTEMI, UA \\
\hline Shah 2014 [15] & Death, bleeding & 9 days, 30 days & UA, NSTEMI \\
\hline Zhao 2015 [12] & Death, MI, major bleeding, minor bleeding, stroke & 7 days, 30 days, 6 months & NSTEMI \\
\hline Zhao 2016 [16] & Death, MI, stroke, major bleeding, all bleeding & 30 days, 1 year & STEMI, NSTEMI, UA \\
\hline Soeiro 2016 [10] & Death, MI, major bleeding, stroke & In-hospital & NSTEMI \\
\hline
\end{tabular}

Abbreviations: MI myocardial infarction, TIMI thrombolysis in myocardial infarction, STEMI ST elevated myocardial infarction, NSTEMI non-ST elevated myocardial infarction, UA unstable angina 
analysis, the PRISMA guideline [5] was followed and the bias risk among the trials was assessed with reference to the features which have been stated in the Cochrane Collaboration [6]. All the trials were rated as having a 'low to moderate' risk of bias.

\section{Statistical analysis}

The statistical analysis was carried out by Revman 5.3 software with odds ratio (OR) and 95\% confidence intervals $(\mathrm{CI})$ as the analytical parameters. Two simple statistical methods were used to assess heterogeneity [7] across the studies namely the $\mathrm{Q}$ statistic test and the $\mathrm{I}^{2}$ test.

A $P$ value of less or equal to 0.05 was considered as statistically significant whereas a $P$ value greater than 0.05 was insignificant in this analysis.

On the other hand, a fixed effects model or a random effects model was used during the analysis depending on the value of $\mathrm{I}^{2}$. A fixed effects model was recommended if an $\mathrm{I}^{2}$ value $<50 \%$ was obtained, whereas if the $\mathrm{I}^{2}$ value was $>50 \%$, a random effects model was recommended.

In addition, this $\mathrm{I}^{2}$ value was also used to predict heterogeneity. The lower the $\mathrm{I}^{2}$ value, the lesser would be the heterogeneity whereas heterogeneity would increase with an increasing $\mathrm{I}^{2}$ value.

Sensitivity analyses were also carried out by excluding each study one by one and a new analysis was carried out each time.

Publication bias was visually observed by analyzing the funnel plots which were generated through the RevMan software. Since this analysis did not involve a large volume of studies (only 7 studies were available), asymmetry of the funnel plots was sufficient to represent publication bias. Normally, if the total number of studies which were included was more than 10 , other methods would have been more appropriate.

Ethical approval was not required for this type of study.

\section{Results}

\section{Search results}

Seven hundred and eighty-seven (787) articles were obtained using the above mentioned searched terms from electronic databases. After a careful check of the abstracts and titles by the same two authors (PKB and MS), 764 articles were eliminated (not related to this current idea). Twenty-three (23) full-text articles were assessed for eligibility. Among these 23 articles, further studies were eliminated because they were:

- Systematic reviews, case studies or letter to editors (2);

- Articles with data which were irrelevant for this current meta-analysis (1);

- Articles which did not report adverse clinical outcomes as their endpoints (4);
- Duplicates or studies which were associated with the same trials or cohorts (9).

Finally, 7 studies were selected and included in this analysis (Fig. 1).

\section{General features of the studies which were included}

Five (5) observational studies and 2 randomized trials were included with a total number of 9618 patients (6587 patients were treated with enoxaparin and 3031 patients were treated with fondaparinux). Regions of patients' enrollment included France, China, Brazil, and Canada with an enrollment period ranging between years 2003 to 2015 . The general features of the studies which were included have been listed in Tables 2 and 3.

\section{Baseline features of the studies which were included}

The baseline features (Table 4) were as follow: mean age 57.3-67.0 years, majority of the patients were male patients, other co-morbidities and smoking history were also reported in both groups. Overall, there were no significant differences in the baseline features between patients who were treated with enoxaparin and fondaparinux.

The OASIS 5 trial had a large number of patients when compared to the other studies. However, in order for the current results not to be influenced by the results obtained in the OASIS 5 trial, the proportion of patients obtained from the OASIS 5 trial was reduced in order to adjust to this current analysis. To be more clear, only the percentage of female patients (20-30\%) from the trial were included when analyzing the 30 -day outcomes and only 11 to $12 \%$ of patients were included when assessing bleeding events. It should be noted that the total number of patients which were extracted was not reduced. For example, ' 10 events of death out of 100 patients' was represented as ' 1 event of death out of 10 patients'. Therefore, it is basically the same thing.

Table 5 summarized other anti-platelet and anticoagulant medications which were used by the patients. It can clearly be seen that almost all the patients were also being treated by aspirin and clopidogrel. Glycoprotein IIb/IIIa, prasugrel and unfractionated heparin were also being used by certain patients.

\section{Less than 10-days follow up period (enoxaparin versus fondaparinux)}

Results of this analysis showed that mortality was similarly observed between enoxaparin and fondaparinux with OR: $1.05,95 \% \mathrm{CI}: 0.67-1.63 ; P=0.84$. MI and stroke were also not significantly different with OR: $0.77,95 \%$ CI: $0.59-1.02 ; P=0.07$ and OR: 1.12 , 95\% CI: $0.51-2.46 ; P=0.78$ respectively during this 10-day period. However, minor, major and total 


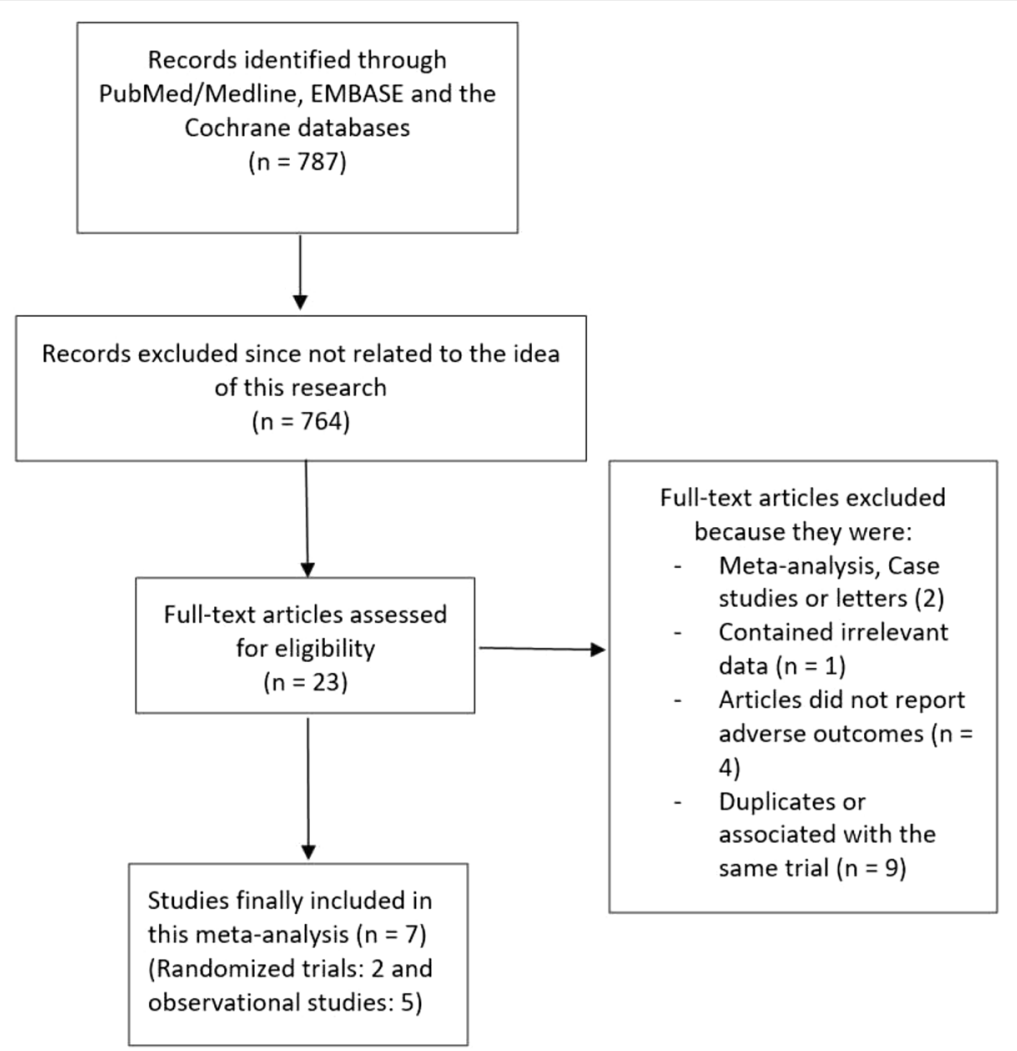

Fig. 1 Flow diagram representing the study selection

bleeding were significantly lower with fondaparinux (OR: 0.40, 95\% CI: 0.27-0.58; $P=0.00001$ ), (OR: 0.46, 95\% CI: $0.32-0.66 ; P=0.0001$ ) and (OR: 0.47 , 95\% CI: $0.37-0.60 ; P=0.00001)$ respectively. Results for this 10-day follow up period have been represented in Fig. 2.

When observational data were separately analyzed, MI and major bleeding significantly favored fondaparinux with OR: $0.61,95 \%$ CI: $0.40-0.94 ; P=0.02$ and OR: 0.44 , 95\% CI: $0.23-0.86 ; P=0.02$ respectively (Fig. 3 ).

However, when randomized data were separately analyzed, mortality was not significantly different between these two drugs with OR: $1.00,95 \%$ CI: $0.53-1.91$; $P=0.99$ whereas total bleeding still significantly favored fondaparinux with OR: $0.40,95 \%$ CI: $0.30-0.55$; $P=0.00001$ (Fig. 4).

\section{0-days follow up period (enoxaparin versus fondaparinux)}

During this 30-days follow up period, mortality and MI were not significantly different with OR: 0.90, 95\% CI: $0.57-1.42$; $P=0.66$ and OR: 1.00 , 95\% CI: $0.69-$ 1.46; $P=1.00$ respectively. However, major and minor bleeding significantly favored fondaparinux with OR:

Table 2 General features of the studies which were included

\begin{tabular}{|c|c|c|c|c|c|}
\hline Studies & Type of study & Region & Patients' enrollment year & $\begin{array}{l}\text { Total no of patients in } \\
\text { Enoxaparin group (n) }\end{array}$ & $\begin{array}{l}\text { Total no of patients in } \\
\text { Fondaparinux group (n) }\end{array}$ \\
\hline FAST MI & Observational & France & 2010 & 1027 & 240 \\
\hline OASIS 5 & $\mathrm{RCT}$ & Canada & $2003-2005$ & 1420 & 1414 \\
\hline Schiele2010 & Observational & France & $2006-2007$ & 1418 & 301 \\
\hline Shah2014 & $\mathrm{RCT}$ & - & 2010 & 90 & 90 \\
\hline Zhao2015 & Observational & China & $2011-2012$ & 232 & 229 \\
\hline Zhao2016 & Observational & China & 2010-2012 & 453 & 422 \\
\hline Soeiro2016 & Observational & Brazil & 2010-2015 & 1947 & 335 \\
\hline Total no of patients (n) & & & & 6587 & 3031 \\
\hline
\end{tabular}


Table 3 Procedures and duration of therapy

\begin{tabular}{|c|c|c|c|c|}
\hline \multirow[t]{2}{*}{ Studies } & Procedures & $\mathrm{PCl}(\%)$ & Type of CAD & Duration of therapy \\
\hline & Eno/Fond & Eno/Fond & Eno/Fond & Eno/Fond \\
\hline FAST MI & $\mathrm{PCl}$ & $69.0 / 69.0$ & NSTEMI & 2 days \\
\hline OASIS 5 & $\mathrm{PCl}$ & $100 / 100$ & NSTEMI, UA & $2-8$ days \\
\hline Schiele2010 & $\mathrm{PCl}$ & $84.0 / 72.0$ & STEMI, NSTEMI, UA & In hospital period \\
\hline Shah2014 & $\mathrm{PCl}$ & $100 / 100$ & UA, NSTEMI & In hospital period \\
\hline Zhao2015 & $\mathrm{PCl}$ & $100 / 100$ & NSTEMI & 2-8 days \\
\hline Zhao2016 & $\mathrm{PCl}$ & $100 / 100$ & STEMI, NSTEMI, UA & 3-7 days \\
\hline Soeiro2016 & $\mathrm{PCl}$ & $100 / 100$ & NSTEMI & In hospital period \\
\hline
\end{tabular}

Abbreviations: PCI percutaneous coronary intervention, Eno enoxaparin, Fond fondaparinux, CAD coronary artery disease, STEMI ST segment elevated myocardial infarction, NSTEMI non-ST segment elevated myocardial infarction, UA unstable angina

0.49, 95\% CI: $0.26-0.94 ; P=0.03$ and OR: $0.48,95 \%$ CI: $0.27-0.85 ; P=0.01$ respectively. Results for the 30-days follow up period have been represented in Fig. 5.

When observational data were separately analyzed, mortality was not significantly different with OR: 0.84 , 95\% CI: 0.44-1.60; $P=0.60$. However, even if major bleeding favored fondaparinux with OR: $0.41,95 \% \mathrm{CI}$ : $0.13-1.31 ; P=0.13$, the result was not statistically significant (Fig. 6).

\section{Midterm follow up period (enoxaparin versus fondaparinux)}

During this midterm follow up period, mortality, MI, and stroke were still not significantly different with OR: 0.79, 95\% CI: 0.50-1.23; $P=0.30$, OR: 1.05 , 95\% CI: $0.78-1.42 ; P=0.73$ and OR: 0.73 , 95\% CI: $0.38-1.41$; $P=0.35$ respectively. However, major, minor and total bleeding were significantly lower with fondaparinux with OR: 0.50, 95\% CI: $0.28-0.89 ; P=0.02$, OR: 0.51, 95\% CI: $0.31-0.84 ; P=0.009$ and OR: $0.48,95 \%$ CI: $0.34-0.69$; $P=0.0001$ respectively (Fig. 7 ).

When observation studies were separately analyzed, mortality, MI and stroke were not significantly different with OR: 0.52, 95\% CI: 0.19-1.39; $P=0.19$, OR: 0.93, 95\% CI: $0.52-1.65$; $P=0.80$ and OR: 0.85 , 95\% CI: $0.33-$ 2.17; $P=0.74$ respectively. However, even if major bleeding favored fondaparinux with OR: $0.43,95 \% \mathrm{CI}$ : $0.16-1.14 ; P=0.09$, the result was not statistically significant but total bleeding was significantly lower with fondaparinux with OR: 0.50, 95\% CI: 0.33-0.78; $P=0.002$ (Fig. 8).

\section{Sensitivity analysis}

Sensitivity analyses were carried out. During the 30-days follow up period, excluding study Zhang2016 resulted in a non-significant result associated with major bleeding with OR: $0.53,95 \%$ CI: $0.27-1.04 ; P=0.06$. During the midterm follow up period, excluding trial OASIS 5 resulted in a non-significant major bleeding with OR: 0.43 , 95\% CI: $0.16-1.14 ; P=0.09$. For the other results, no significant change was observed.

In addition, when patients with STEMI were excluded, and an analysis was conducted based on patients with NSTEMI, major and minor bleeding were still significantly higher with enoxaparin, with OR: 0.46, 95\% CI: 0.32-0.66; $P=0.0001$ and OR: 0.40 , 95\% CI: $0.27-0.58$; $P=0.00001$ respectively during a 10-day follow up period. During a 30-day follow up period, only minor bleeding was significantly higher with enoxaparin, with OR: $0.48,95 \%$ CI: $0.27-0.85 ; P=0.01$. In addition, during the mid-term follow up, major and minor bleeding were both significantly higher with enoxaparin, with OR: 0.55, 95\% CI: 0.30-0.99; $P=0.05$ and OR: $0.51,95 \% \mathrm{CI}$ :

Table 4 Baseline features of the studies which were included

\begin{tabular}{|c|c|c|c|c|c|c|}
\hline \multirow[t]{2}{*}{ Studies } & Mean age & Males (\%) & $\mathrm{Ht}(\%)$ & Ds (\%) & Cs (\%) & DM (\%) \\
\hline & $E / F$ & $\mathrm{E} / \mathrm{F}$ & $E / F$ & $\mathrm{E} / \mathrm{F}$ & $E / F$ & $E / F$ \\
\hline FAST MI & $67.0 / 66.5$ & 71.0/72.0 & $58.0 / 66.0$ & $45.5 / 52.5$ & $27.0 / 32.0$ & $23.0 / 25.0$ \\
\hline OASIS 5 & $64.5 / 64.6$ & 69.1/71.7 & - & - & - & $23.1 / 23.5$ \\
\hline Schiele2010 & - & $71.0 / 66.0$ & $52.0 / 57.0$ & $49.0 / 55.0$ & $32.0 / 31.0$ & $21.0 / 20.0$ \\
\hline Shah2014 & - & 77.8/74.4 & $55.6 / 55.6$ & - & $58.9 / 55.6$ & $41.1 / 38.9$ \\
\hline Zhao2015 & $59.8 / 60.1$ & 73.3/76.9 & $65.5 / 66.8$ & - & $68.5 / 68.1$ & $39.6 / 37.1$ \\
\hline Zhao2016 & $58.2 / 57.3$ & 78.2/76.3 & $59.5 / 57.2$ & - & $53.0 / 52.1$ & $20.3 / 22.8$ \\
\hline Soeiro2016 & $61.8 / 61.0$ & $62.6 / 65.7$ & $73.6 / 67.8$ & $51.2 / 48.9$ & $30.5 / 24.2$ & $46.9 / 55.8$ \\
\hline
\end{tabular}


Table 5 Other anti-platelet and anticoagulant medications which were used by the participants

\begin{tabular}{|c|c|c|c|c|c|}
\hline \multirow[t]{2}{*}{ Studies } & Aspirin & Clopidogrel & Glycoprotein IIb/IIla & Prasugrel & U.heparin \\
\hline & $\mathrm{E} / \mathrm{F}$ & $\mathrm{E} / \mathrm{F}$ & $\mathrm{E} / \mathrm{F}$ & $E / F$ & $\mathrm{E} / \mathrm{F}$ \\
\hline FAST MI & $98.0 / 98.0$ & $90.5 / 92.0$ & $26.5 / 34.0$ & $15.0 / 15.0$ & $36.0 / 60.0$ \\
\hline OASIS 5 & $98.9 / 98.6$ & $92.3 / 91.1$ & $38.8 / 40.4$ & - & - \\
\hline Schiele2010 & $99.0 / 99.0$ & $98.0 / 99.0$ & $58.0 / 68.0$ & - & - \\
\hline Zhao2015 & $96.5 / 96.1$ & $100 / 100$ & - & - & $100 / 100$ \\
\hline Zhao2016 & $99.8 / 99.3$ & $75.3 / 80.1$ & - & - & - \\
\hline Soeiro2016 & $97.8 / 98.5$ & $67.9 / 65.4$ & $16.1 / 5.80$ & - & - \\
\hline
\end{tabular}

Abbreviations: $E$ enoxaparin, $F$ fondaparinux, data were represented in terms of $\%$

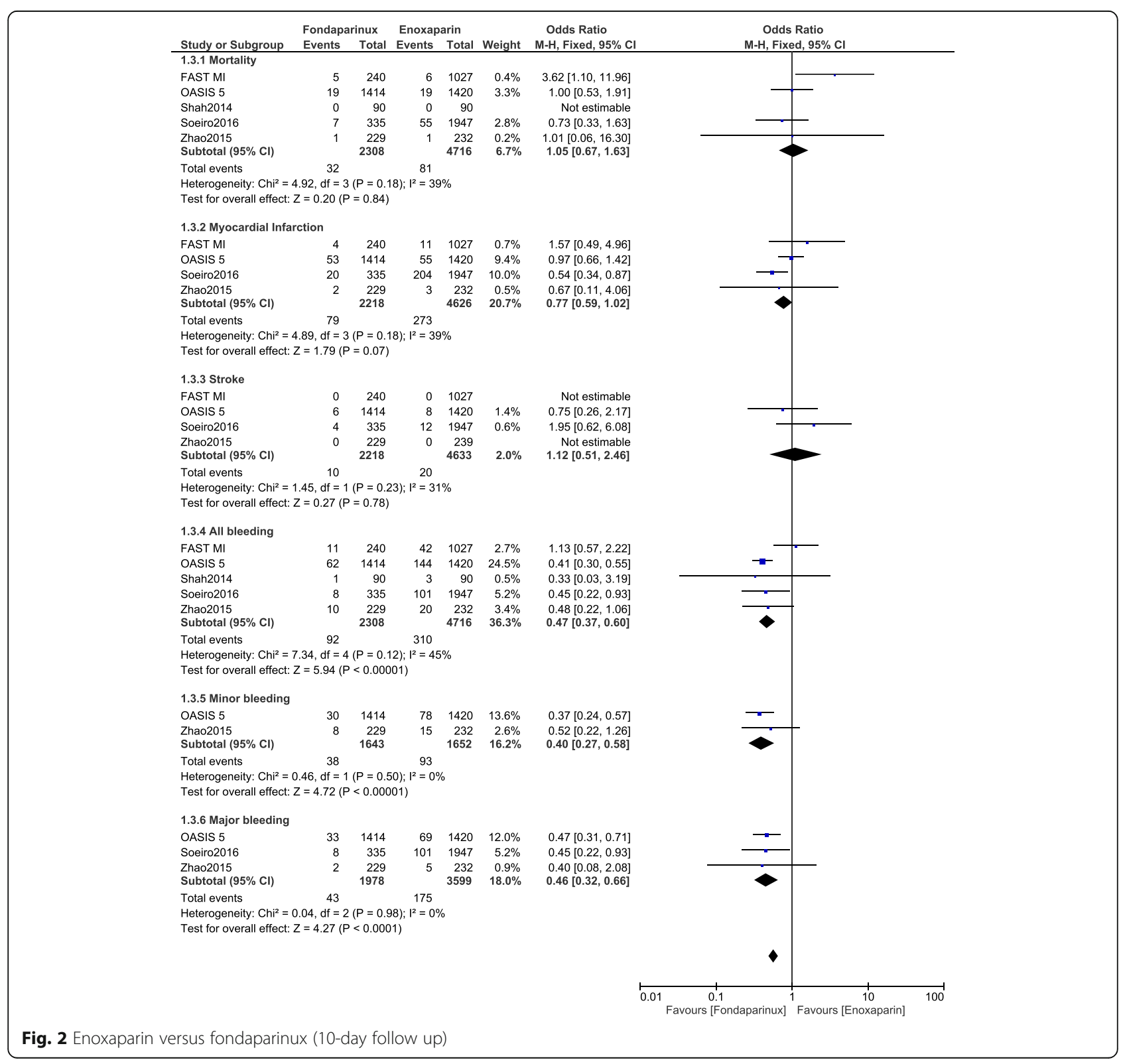




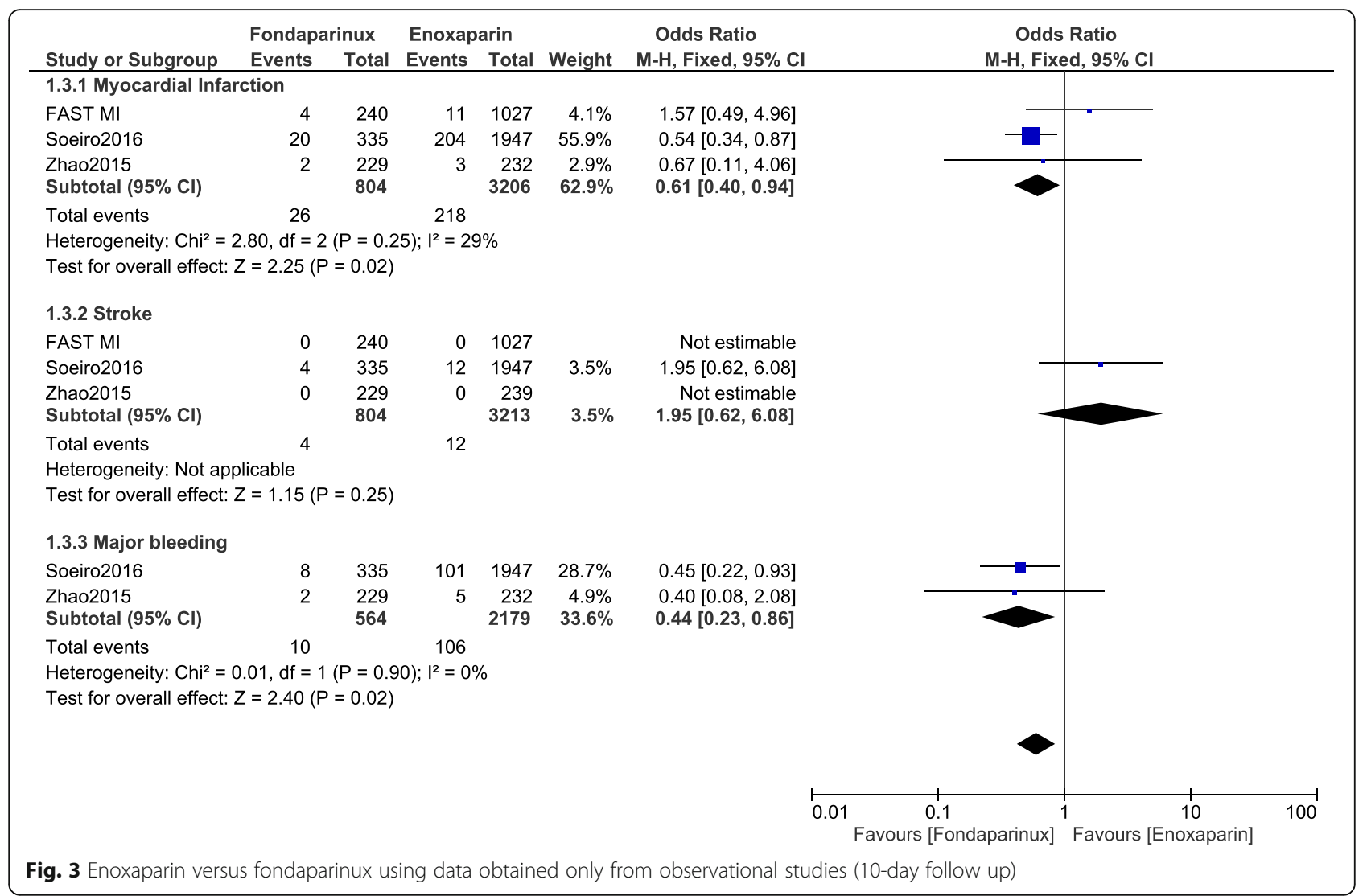

0.31-0.84; $P=0.009$ respectively in these patients with NSTEMI.

\section{Publication bias}

This current analysis included of a total number of 7 studies ( 2 randomized and 5 observational studies) that assessed all the clinical endpoints with minimal evidence of publication bias which was visually observed through the funnel plots obtained (Figs. 9, 10, 11).

These funnel plots represented minimal publication bias. Possible reasons might be the presence of

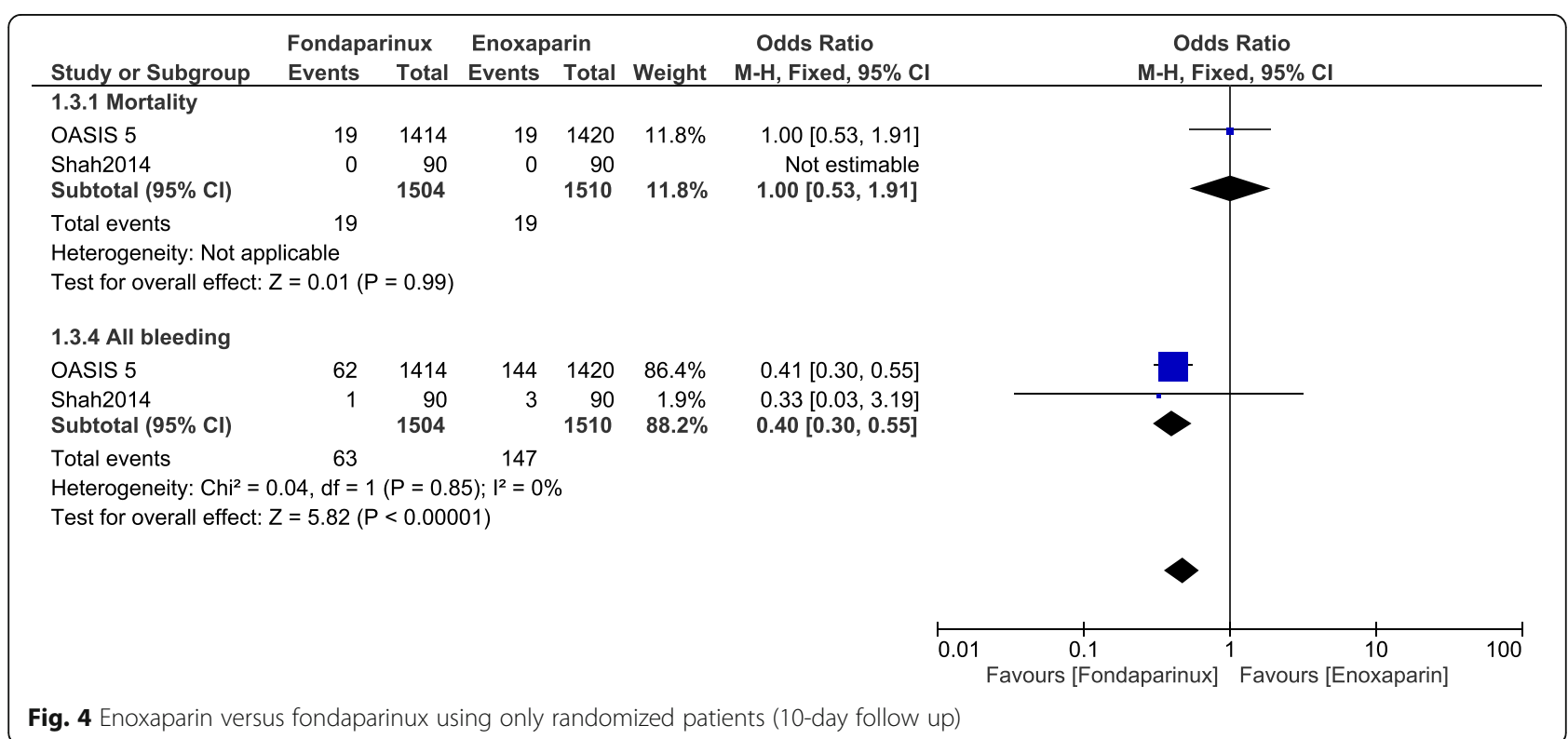




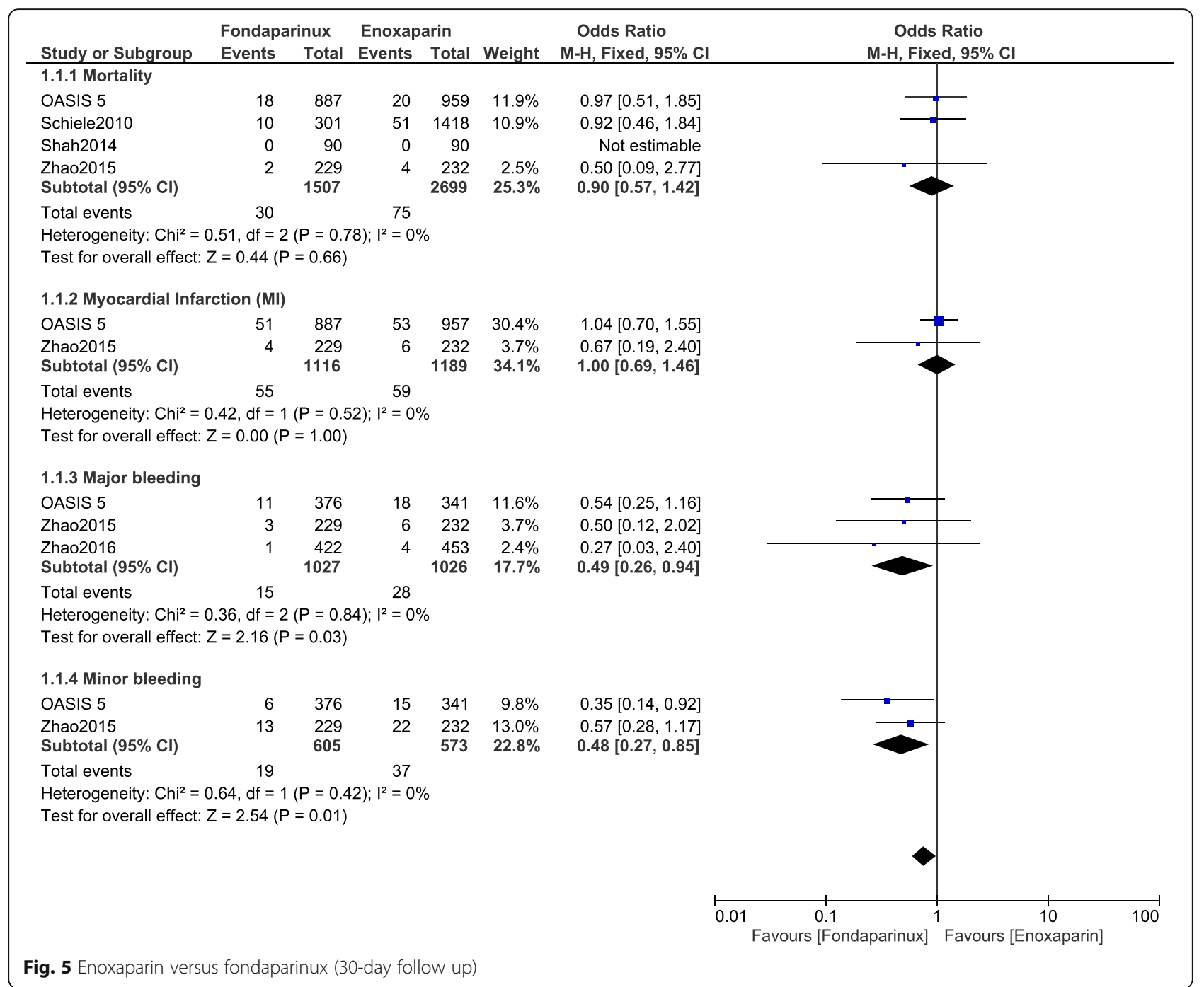

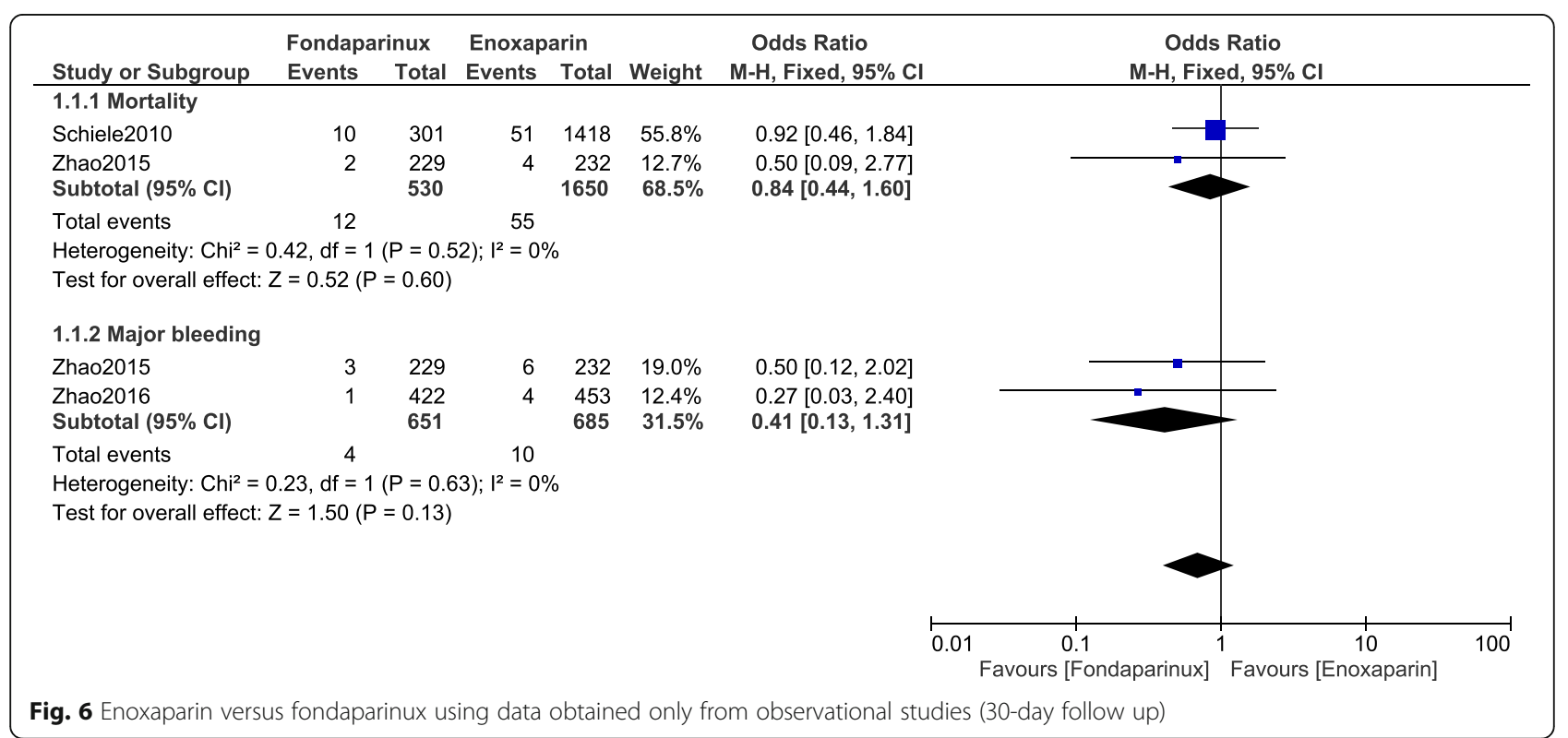




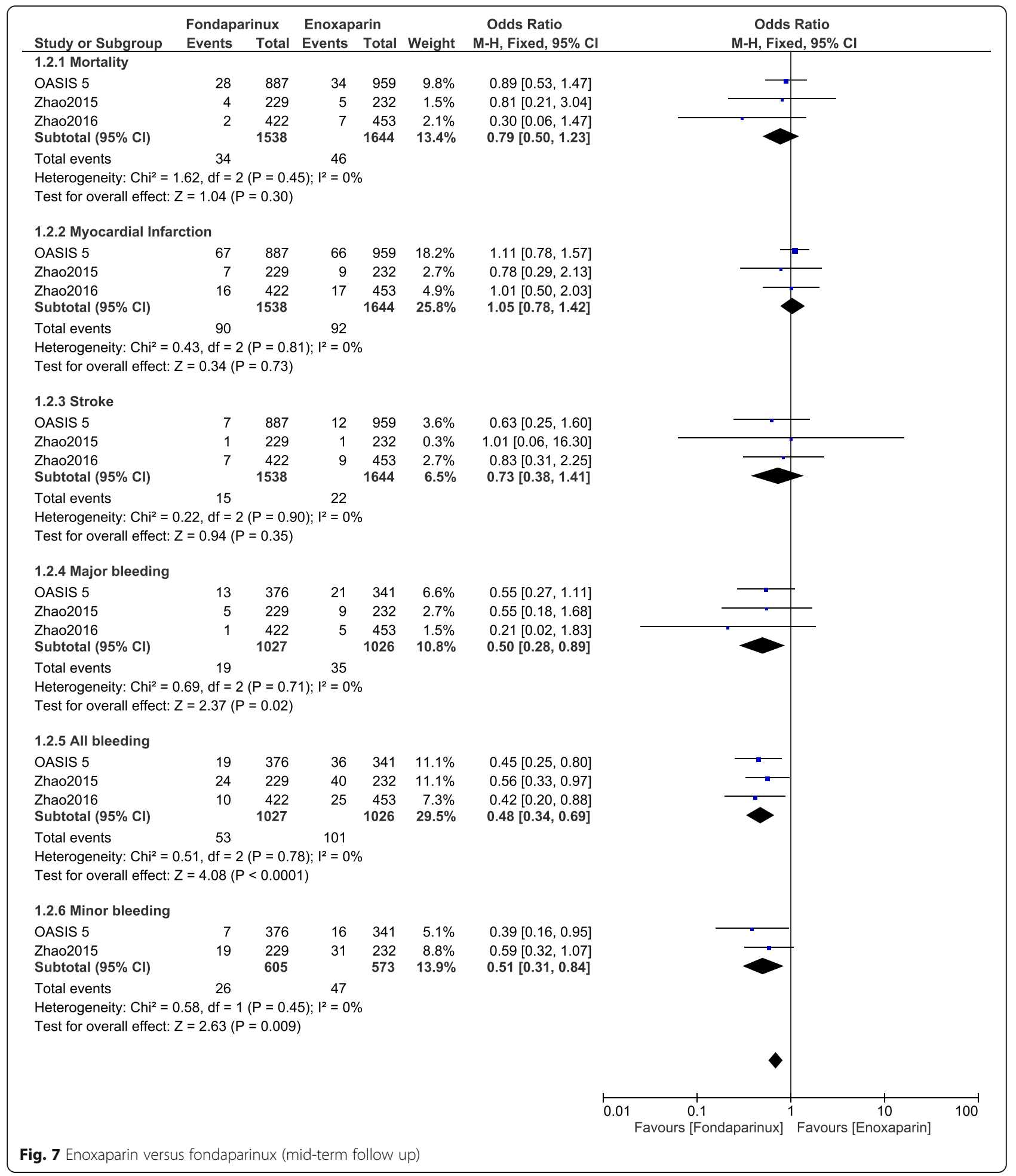

observational studies, and the selection of only English publications for this analysis.

\section{Discussion}

This analysis aimed to compare enoxaparin with fondaparinux. Current results showed both anticoagulants to have similar mortality and stroke rates. However, fondaparinux was associated with a significantly lower major and minor bleeding when compared to enoxaparin in majority of the bleeding subgroups which were analyzed.

Surprisingly, even if the use of glycoprotein IIb/IIIa was more in patients who used fondaparinux, a low 


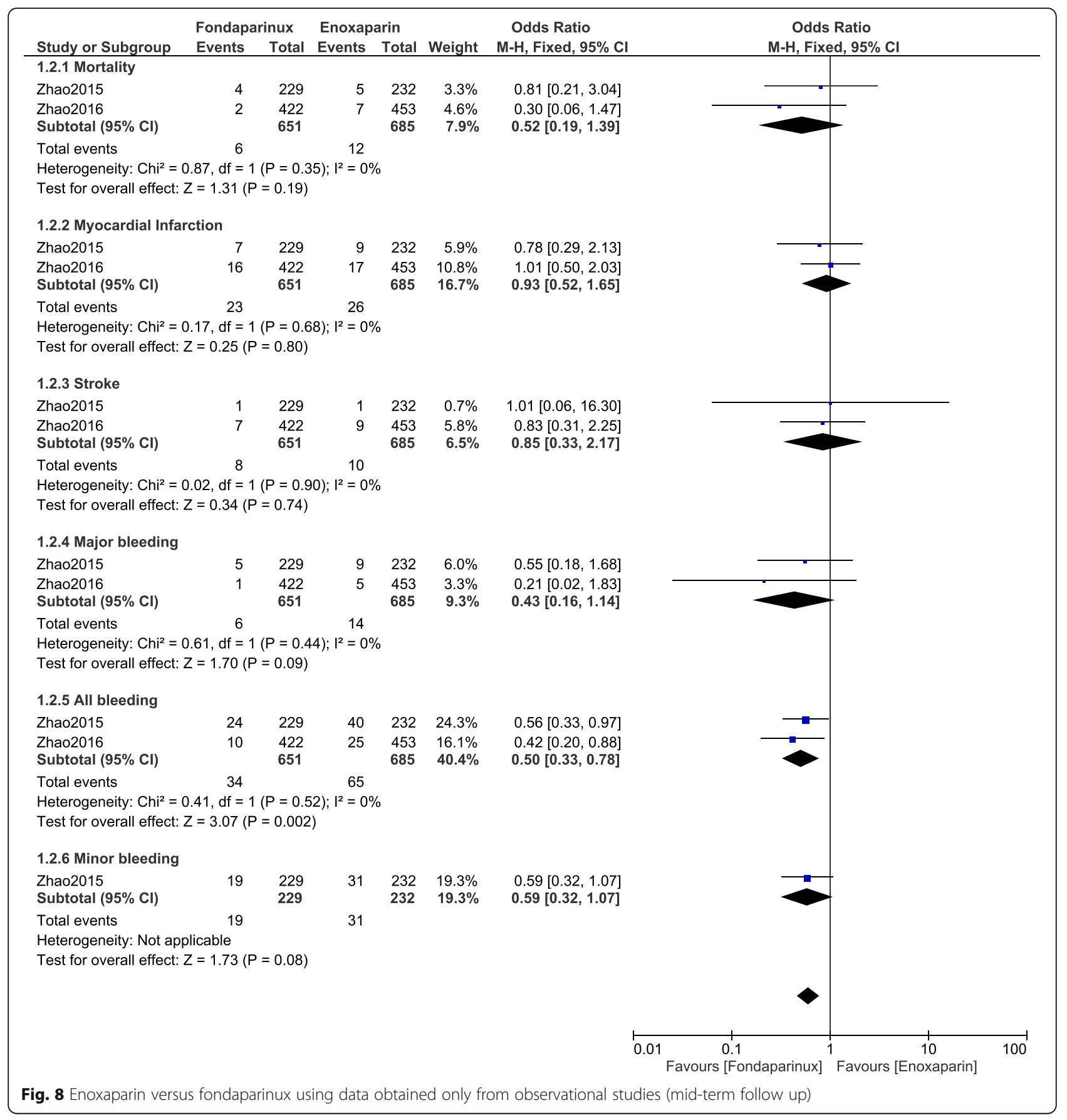

bleeding was observed with this anticoagulant when compared to enoxaparin.

At this stage, it would be interesting to know the mechanisms of action of fondaparinux and enoxaparin. Fondaparinux is a factor Xa inhibitor and does not inhibit thrombin (IIa) [8]. Enoxaparin on the other hand, binds to antithrombin to form a complex molecule that can irreversibly inactivate clotting factor $\mathrm{Xa}$ and it has less activity against thrombin [9]. This is how these two anticoagulants work.
The Brazilian Registry Data which was a multicentered retrospective observational study including 2282 patients also supported the result of this current analysis which is similar to recently published data in international literature showing the superiority of fondaparinux to enoxaparin in the Brazilian population in terms of bleeding outcomes following PCI $(40.1 \%$ of the patients in the fondaparinux group and $35.1 \%$ of patients in the enoxaparin group underwent PCI) [10]. 


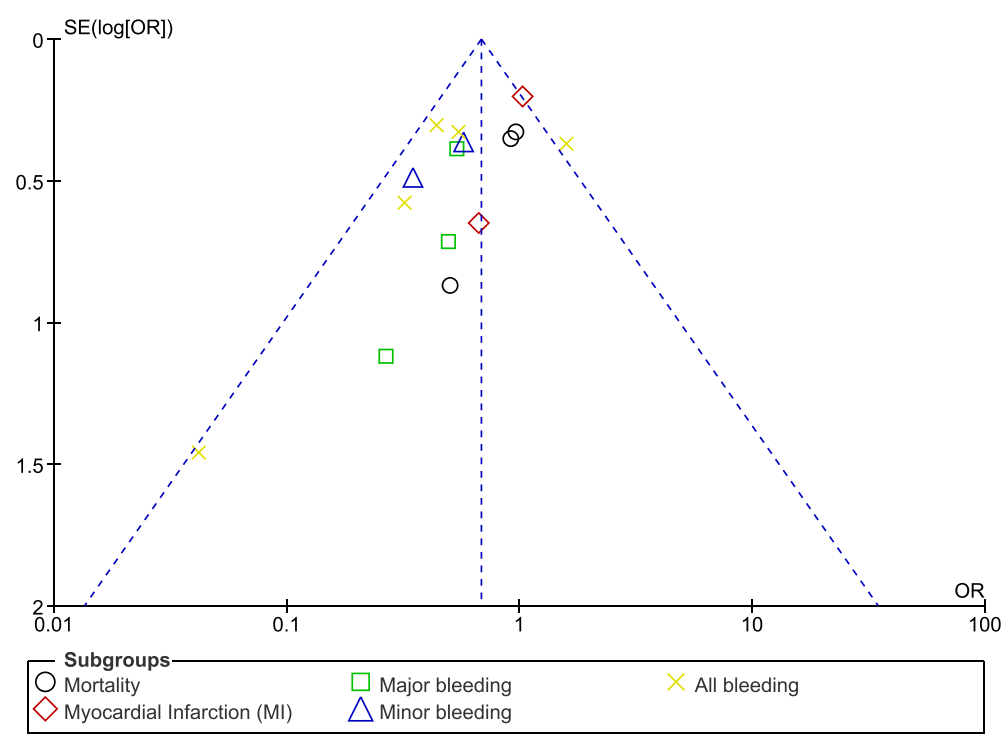

Fig. 9 Funnel plot representing publication bias

Similarly, the OASIS 5 trial which was a double blinded randomized trial comparing fondaparinux with enoxaparin in 6238 patients who underwent PCI showed reduced bleeding events to be associated with fondaparinux without any increase in mortality [2].

On the other hand, the OASIS 6 trial (12,092 patients obtained from 447 hospitals in 41 countries around the globe) which compared fondaparinux with placebo or unfractionated heparin in patients with STEMI showed that in patients who were not undergoing PCI, the former was associated with a lower mortality and reinfarction without increasing stroke or bleeding events
[11]. However, our analysis showed no significant difference in mortality or stroke, but with significantly lower bleeding events following invasive procedures when fondaparinux was compared to enoxaparin. Could it be the invasive procedure which contributed to these different results obtained from OASIS 5 and 6 ?

To provide an answer to this question, a small Chinese study with NSTEMI patients who underwent PCI, showed no statistically significant difference in bleeding outcomes between fondaparinux and enoxaparin. However, it should not be ignored that both groups were also additionally treated with tirofiban [12] and the total

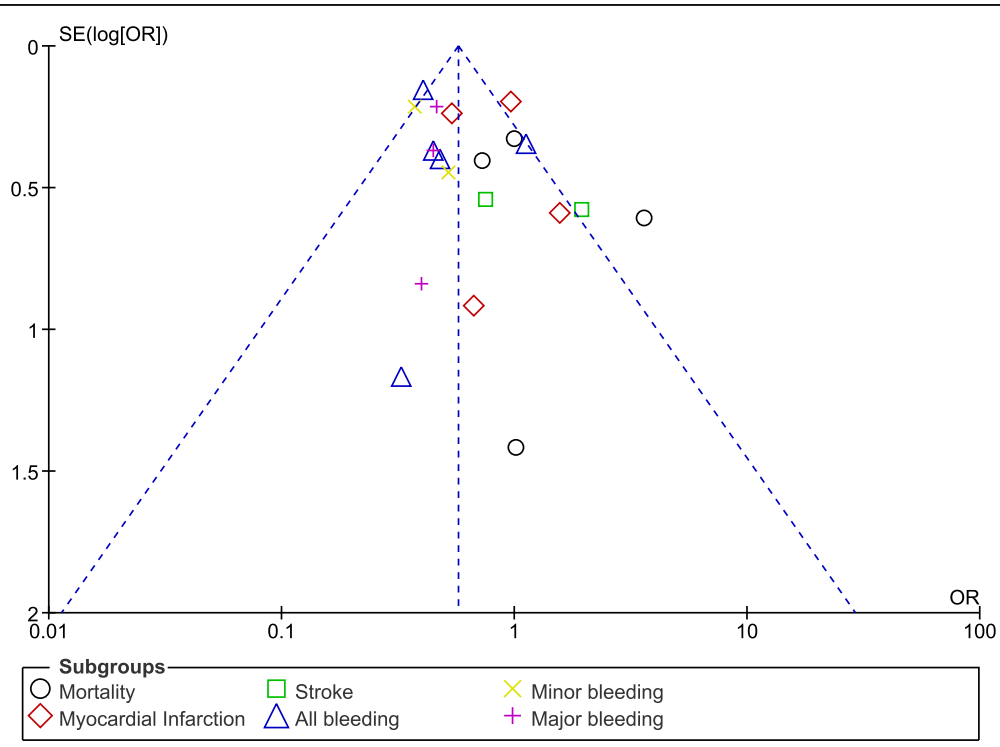

Fig. 10 Funnel plot representing publication bias 


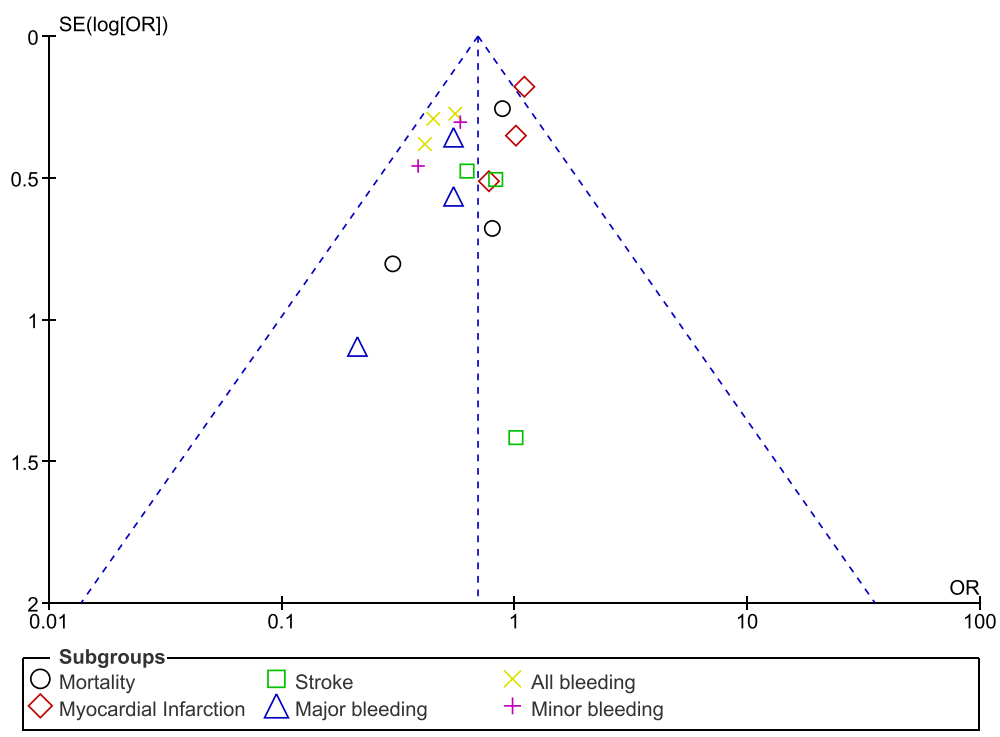

Fig. 11 Funnel plot representing publication bias

number of patients enrolled was less when compared to the OASIS 5 and 6.

Moreover, the French cohort of NSTEMI patients who were predominantly managed invasively did not show fondaparinux to be superior to enoxaparin in terms of bleeding outcomes [3]. However, similar to the results of this analysis, mortality was not significantly different between these two anticoagulants. Nevertheless, it was shown that during the propensity score matched cohorts, a lower number of patients used aspirin or clopidogrel before admission.

Finally, the fact that several factors such as comorbidities, age, use of anti-platelets and anticoagulants, as well as the dosage and whether these blood thinning agents were used before and after admission and the effect of plaque should all be taken into consideration when assessing bleeding risk and other adverse clinical outcomes in such patients. For example, a recent metaanalysis showed thin-cap fibro-atheroma to be highly associated with culprit plaque rupture [13]. This prevalence was higher in patients with STEMI compared to patients with NSTEMI or unstable angina. Therefore, clinical outcomes might vary from study to study due to the influence of such factors.

\section{Novelty}

This analysis is new in several ways:

- It is among the first meta-analyses comparing enoxaparin with fondaparinux in patients who were being treated for ACS.

- This interesting idea is very important clinically.
- A low to moderate level of heterogeneity was observed in several of the subgroups analyzing the outcomes, which might be a positive aspect of this analysis.

- Randomized patients and patients obtained from observational studies were combined as well as separately analyzed.

\section{Limitations}

Limitations were as follow:

- Due to the limited number of patients analyzed, the results might not be very accurate.

- This analysis concerned mainly patients suffering from NSTEMI.

- Classification of bleeding events was vast. Bleeding should be classified as TIMI defined bleeding, BARC defined bleeding and ACUITY defined bleeding and then analyzed.

- Use of other anti-platelets and anticoagulants and other factors might have influenced the results which were obtained.

\section{Conclusion}

In patients who were treated for ACS, fondaparinux might be a better choice when compared to enoxaparin in terms of short to midterm bleeding events. This result was mainly applicable to patients with NSTEMI. However, due to a limited number of patients analyzed, further larger randomized trials should be able to confirm this hypothesis. 


\section{Abbreviations}

ACS: acute coronary syndrome; RCTs: randomized controlled trials; $\mathrm{PCl}$ : percutaneous coronary intervention

\section{Acknowledgement}

Not applicable.

\section{Funding}

There was no external source of funding for this research.

\section{Availability of data and materials}

All data and materials used in this research are freely available. References have been provided.

\section{Authors' contributions}

PKB, MS and JY were responsible for the conception and design, acquisition of data, analysis and interpretation of data, drafting the initial manuscript and revising it critically for important intellectual content. PKB wrote this manuscript. All authors read and approved the final manuscript.

\section{Competing interests}

The authors declare that they have no competing interests.

\section{Consent for publication}

Not applicable.

\section{Ethics approval and consent to participate}

Ethical approval was not applicable for this systematic review and metaanalysis.

\section{Publisher's Note}

Springer Nature remains neutral with regard to jurisdictional claims in published maps and institutional affiliations.

\section{Author details}

${ }^{1}$ Institute of Cardiovascular Diseases, the First Affiliated Hospital of Guangxi Medical University, Nanning, Guangxi 530027, People's Republic of China. ${ }^{2}$ Department of Paediatrics, Pragati Children's Hospital, Ch Pet, Andhra Pradesh, India. ${ }^{3}$ Department of Cardiology, The People's Hospital of Guangxi Zhuang Autonomous Region, Nanning, Guangxi 530021, China.

Received: 28 February 2017 Accepted: 2 May 2017

Published online: 08 May 2017

\section{References}

1. Weber K, Spirk D, Pieper M, Kucher N. Clinical efficacy and safety of enoxaparin in unselected Swiss patients undergoing primary or elective percutaneous coronary intervention: analysis of the RIVIERA study. Acta Cardiol. 2009;64(4):455-9.

2. Mehta SR, Granger CB, Eikelboom JW, Bassand JP, Wallentin L, Faxon DP, Peters RJ, Budaj A, Afzal R, Chrolavicius S, Fox KA, Yusuf S. Efficacy and safety of fondaparinux versus enoxaparin in patients with acute coronary syndromes undergoing percutaneous coronary intervention: results from the OASIS-5 trial. J Am Coll Cardiol. 2007;50(18):1742-51.

3. Puymirat E, Schiele F, Ennezat PV, Coste P, Collet JP, Bonnefoy-Cudraz E, Roul G, Richard P, Simon T, Danchin N. Impact of fondaparinux versus enoxaparin on in-hospital bleeding and 1-year death in non-STsegmentelevation myocardial infarction. FAST-MI (French Registry of acute ST-elevation and non-ST-elevation myocardial infarction) 2010. Eur Heart J Acute Cardiovasc Care. 2015;4(3):211-9.

4. The Thrombolysis in Myocardial Infarction (TIMI) trial. Phase I findings. TIMI Study Group. N Engl J Med. 1985. 4;312(14):932-6.

5. Liberati A, Altman DG, Tetzlaff J, et al. The PRISMA statement for reporting systematic reviews and meta-analyses of studies that evaluate healthcareinterventions: explanation and elaboration. BMJ. 2009;339:b2700

6. Higgins JP, Thompson SG, Deeks JJ, et al. Measuring inconsistency in metaanalyses. BMJ. 2003;327:557-60

7. Higgins JPT, Thompson SG, Deeks JJ, Altman DG. Measuring inconsistency in meta-analyses. BMJ. 2003;327(7414):557-60.

8. Bauer KA. Fondaparinux sodium: a selective inhibitor of factor Xa. Am J Health Syst Pharm. 2001;58(Suppl 2):S14-7.
9. Yau JW, Stafford AR, Liao P, Fredenburgh JC, Roberts R, Weitz JI. Mechanism of catheter thrombosis: comparison of the antithrombotic activities of fondaparinux, enoxaparin, and heparin in vitro and in vivo. Blood. 2011; 118(25):6667-74.

10. Soeiro AM, Silva PG, Roque EA, Bossa AS, César MC, Simões SA, Okada MY, Leal TC, Pedroti FC, Oliveira MT Jr. Fondaparinux versus Enoxaparin - Which is the Best Anticoagulant for Acute Coronary Syndrome? - Brazilian Registry Data. Arq Bras Cardiol. 2016;107(3):239-244.

11. Yusuf S, Mehta SR, Chrolavicius S, Afzal R, Pogue J, Granger CB, Budaj A, Peters RJ, Bassand JP, Wallentin L, Joyner C, Fox KA, OASIS-6 Trial Group. Effects of fondaparinux on mortality and reinfarction in patients with acute ST-segment elevation myocardial infarction: the OASIS-6 randomized trial. JAMA. 2006;295(13):1519-30.

12. Zhao XM, Gao CY, Chu YJ, Yang L, Yang XZ, Xu WK, He WQ, Zhang PR, Liu $X Y$, Tian LX. FondaparinuX vs. enoxaparin in patients with non-ST elevation acute coronary syndromes (NSTE-ACS) treated with percutaneous coronary intervention and tirofiban: an exploratory study in China. J Clin Pharm Ther. 2015.

13. lannaccone M, Quadri G, Taha S, et al. Prevalence and predictors of culprit plaque rupture at OCT in patients with coronary artery disease: a metaanalysis. Eur Heart J Cardiovasc Imaging. 2016;17(10):1128-37.

14. Schiele F, Meneveau N, Seronde MF, Descotes-Genon V, Dutheil J, Chopard R, Ecarnot F, Bassand JP, Reseau de Cardiologie de Franche Comte. Routine use of fondaparinux in acute coronary syndromes: a 2-year multicenter experience. Am Heart J. 2010;159(2):190-8.

15. Shah S, Khajuria V Tandon VR, Gillani ZH, Lal M. Comparative evaluation of efficacy, safety and haemostatic parameters of enoxaparin and fondaparinuxin unstable coronary artery disease. J Clin Diagn Res. 2014;8(1):31-4.

16. Zhao X, Yang XX, Ji SZ, Wang XZ, Wang L, Gu CH, Ren LL, Han YL. Efficacy and safety of fondaparinux versus enoxaparin in patients undergoing percutaneous coronaryintervention treated with the glycoprotein IIb/Illa inhibitor tirofiban. Mil Med Res. 2016;3:13

\section{Submit your next manuscript to BioMed Central and we will help you at every step:}

- We accept pre-submission inquiries

- Our selector tool helps you to find the most relevant journal

- We provide round the clock customer support

- Convenient online submission

- Thorough peer review

- Inclusion in PubMed and all major indexing services

- Maximum visibility for your research

Submit your manuscript at www.biomedcentral.com/submit
Biomed Central 